\title{
The cost of hydrocephalus: a cost-effectiveness model for evaluating surgical techniques
}

\author{
Jaims Lim, MD,,2 Alan R. Tang, BA, ,,3 Campbell Liles, BS, 1,2 Alexander A. Hysong, BA, 1,2 \\ Andrew T. Hale, MS, ${ }^{1,2}$ Christopher M. Bonfield, MD, ${ }^{1,4}$ Robert P. Naftel, MD, ${ }^{1,4}$ \\ John C. Wellons III, MD, MSPH, ${ }^{1,4}$ and Chevis N. Shannon, MBA, MPH, DrPH ${ }^{1,4}$
}

${ }^{1}$ Surgical Outcomes Center for Kids, Monroe Carell Jr. Children's Hospital at Vanderbilt; ${ }^{2}$ Vanderbilt University School of Medicine; ${ }^{3}$ Vanderbilt University; and ${ }^{4}$ Department of Neurological Surgery, Vanderbilt University Medical Center, Nashville, Tennessee

OBJECTIVE Many studies have aimed to determine the most clinically effective surgical intervention for hydrocephalus. However, the costs associated with each treatment option are poorly understood. In this study, the authors conducted a cost-effectiveness analysis, calculating the incremental cost-effectiveness ratio (ICER) of ventriculoperitoneal shunting (VPS), endoscopic third ventriculostomy (ETV), and ETV with choroid plexus cauterization (ETV/CPC) in an effort to better understand the clinical effectiveness and costs associated with treating hydrocephalus.

METHODS The study cohort includes patients under the age of 18 who were initially treated for hydrocephalus between January 2012 and January 2015 at the authors' institution. Overall treatment costs were calculated using patient-level hospitalization costs and professional fees reimbursable to the hospital and directly related to the initial and follow-up (postoperative day 1 to 12 months) treatment of hydrocephalus. TreeAge Pro was used to conduct the cost-effectiveness analyses.

RESULTS A total of 147 patients were identified. Based on the initial intervention for hydrocephalus, their cases were classified as follows: 113 VPS, 14 ETV, and 20 ETV/CPC. During the initial intervention, VPS patients required a longer length of stay at 5.6 days, compared to ETVICPC ( 3.35 days) and ETV ( 2.36 days) patients. Failure rates for all treatment options ranged from $29 \%$ to $45 \%$, leading to recurrent hydrocephalus and additional surgical intervention between postoperative day 1 and 12 months. Cost-effectiveness analyses found ETV to be less costly and more clinically effective, with an ICER of $\$ 94,797$ compared to VPS $(\$ 130,839)$ and ETVICPC $(\$ 126,394)$. However, when stratified by etiology, VPS was found to be more clinically effective and cost-effective in both the myelomeningocele and posthemorrhagic hydrocephalus patient groups with an incremental cost per clinical unit of effectiveness (success or failure of intervention) of $\$ 76,620$ compared to ETV and ETV/CPC. However, when assessing cases categorized as "other etiologies," ETV was found to be more costeffective per clinical unit, with an ICER of $\$ 60,061$ compared to ETVICPC $(\$ 93,350)$ and VPS $(\$ 142,135)$.

CONCLUSIONS This study is one of the first attempts at quantifying the patient-level hospitalization costs associated with surgical management of hydrocephalus in pediatric patients treated in the United States. The results indicate that the conversation regarding CSF diversion techniques must be patient-specific and consider etiology as well as any previous surgical intervention. Again, these findings are short-run observations, and a long-term follow-up study should be conducted to assess the cost of treating hydrocephalus over the lifetime of a patient.

https://thejns.org/doi/abs/10.3171/2018.6.PEDS17654

KEYWORDS hydrocephalus; cost-effectiveness; CSF diversion; ventriculoperitoneal shunting; endoscopic third ventriculostomy; choroid plexus cauterization

$\mathrm{H}$ YDROCEPHALUS is the most common pediatric neurosurgical condition, and cerebrospinal fluid (CSF) diversion procedures accrue a total cost of $\$ 2$ billion for hospitals nationwide. ${ }^{17}$ The 3 main corrective procedures performed to treat hydrocephalus are ventriculoperitoneal shunting (VPS) and endoscopic third ventriculostomy (ETV), with or without choroid plexus cauterization (CPC). VPS was first described as a treatment for hydrocephalus in 1955 by Scott, Wycis, Murtagh, and Reyes. ${ }^{14}$ Since then, many studies have aimed to evalu-

ABBREVIATIONS CPC = choroid plexus cauterization; CSF = cerebrospinal fluid; DALY = disability-adjusted life year; ETV = endoscopic third ventriculostomy; ICER = incremental cost-effectiveness ratio; NICU = neonatal intensive care unit; $\mathrm{PHH}=$ posthemorrhagic hydrocephalus; $\mathrm{QALY}=$ quality-adjusted life year; $\mathrm{TCH}=$ total costs of hospitalization; VP = ventriculoperitoneal; VPS = VP shunting.

SUBMITTED November 27, 2017. ACCEPTED June 14, 2018.

INCLUDE WHEN CITING Published online October 5, 2018; DOI: 10.3171/2018.6.PEDS17654. 
ate its clinical effectiveness and to identify factors associated with shunt failure. Although limited in nature, several studies have also assessed the costs associated with VPS and VPS failures in the pediatric hydrocephalus population. ${ }^{4,13,15,16}$ Shunt failure most commonly occurs in children under the age of 1 year, at a rate ranging from $12 \%$ to $30 \%,{ }^{1}$ and accounts for over $\$ 1$ billion in hospital admission costs. ${ }^{16}$ The cost associated with VPS for each patient was found to vary greatly due to underlying comorbidities and medical status, ${ }^{12}$ and the costs associated with different clinical outcomes after VPS are not well defined.

VPS continued to be the primary treatment of hydrocephalus until the mid-1990s, when ETV became a widely acceptable procedure and alternative to the implanted ventriculoperitoneal (VP) shunt. ${ }^{11}$ The Hydrocephalus Clinical Research Network's prospective study on ETV outcomes reported failure rates ranging from $24 \%$ to 45\%. More specifically, based on the ETV Success Score, patients with a score $\geq 80$ had a 6-month Kaplan-Meier success rate of $75.9 \% .^{9}$ Complications following an ETV include CSF leak, hyponatremia, pseudomeningocele, seizure, meningitis, intraventricular hemorrhage, and wound infection.

Several studies have aimed to determine whether VPS or ETV is a better treatment for hydrocephalus. Vinchon et al. ${ }^{18}$ conducted an extensive analysis of factors that affect outcomes following VPS and ETV and identified young age at surgery, prematurity, infection, and spina bifida as some confounding conditions that increased the risk of operational failure for both VPS and ETV. In another study, Drake et al. ${ }^{5}$ found that ETV resulted in a higher net quality-adjusted life year (QALY) score 12 months after surgery than VPS.

In 2007, Dr. Warf evaluated his experience utilizing the combination of endoscopic third ventriculostomy and choroid plexus cauterization (ETV/CPC) and brought attention to this ETV/CPC as a potentially and more clinically effective treatment for pediatric hydrocephalus. ${ }^{19}$ Since that time, ETV/CPC has become more widely adopted by many institutions as the initial treatment intervention for patients with hydrocephalus.

To date, there have been no direct cost comparison or effectiveness studies comparing VPS and ETV as corrective procedures for hydrocephalus in the United States. Investigators in Canada and Brazil (in 2001 and 2014, respectively) conducted cost analyses for ETV, and both studies found no differences in costs when comparing ETV procedures to other CSF diversion procedures. ${ }^{6,10}$ Since the patient population and healthcare infrastructure of the United States is different from that of other countries, such as Brazil and Canada, further investigation is warranted. A systematic evaluation of hydrocephalus etiology and long-term financial impact of hydrocephalus treatment is critically important due to the overall rise in healthcare expenditures in the United States and is therefore the aim of this study.

\section{Methods \\ Data Collection}

All consecutive patients who underwent initial VPS,
ETV, or ETV/CPC procedures between January 2012 and January 2015 at Monroe Carell Jr. Children's Hospital at Vanderbilt University were identified from Vanderbilt's electronic medical records system utilizing ICD-10 codes pertaining to hydrocephalus. Codes included were G91, Q03, Q05, and Q07, along with their respective suffix modifiers and ICD-9 counterparts. Comprehensive ICD and CPT codes used to identify the patient cohort are further detailed in Appendix 1.

Patient demographic and financial data, including costs directly related to the hospitalization associated with the initial permanent CSF diversion, hospital admission, professional fees, and outpatient follow-up visits, were collected from the electronic health record and complemented with clinical, surgical, and follow-up data for our specified study period. Follow-up information after initial permanent CSF diversion was collected up to 12 months after the initial procedure discharge in order to track failure rates and number of postprocedure readmissions. If the patient, having undergone permanent intervention, was found to have treatment failure within a 12-month postoperative period, additional clinical data regarding any new hospitalization entailing surgical intervention were also collected and accounted for. To ensure accurate patient-level clinical data collection and cost calculations, 2 contributing authors (J.L. and A.R.T.) conducted individual chart and cost data reviews for the entire cohort. For the purposes of this analysis, only costs related specifically to neurosurgical care were included. All cost data were retrieved from the financial accounting systems by the Vanderbilt University Medical Center Finance Office and stored in a RedCap database. ${ }^{8}$

\section{Variable Definitions and Charge Calculations}

The mean length of hospitalization, mean daily hospitalization costs, and mean professional procedural fees were obtained from our patient cohort data. To account for inflation across years, all costs were standardized to the year 2015 utilizing the CCEMG-EPPI-Centre Cost Converter (https://eppi.ioe.ac.uk/costconversion/).

For the purposes of this study, the following definitions and calculations were utilized:

Due to the potential for increased length of stay unrelated to neurosurgical issues, we defined length of hospitalization as date of admission to the neonatal intensive care unit (NICU) with neurosurgical consultation or date of neurosurgical intervention to date of hospital discharge or date of neurosurgery sign-off.

Procedure failure was defined as the need for additional neurosurgical intervention, due to either infectious or noninfectious cause, based on clinical symptoms and radiographic changes, and recurring hydrocephalus from initial postoperative day 1 to 12 months after the procedure. The decision for additional surgical intervention was based on neurosurgical evaluation by a neurosurgical resident and attending physician, radiographic evidence of recurrent hydrocephalus (i.e., enlarged ventricles), and symptoms of increased intracranial pressure. Procedure success was defined as no additional required neurosurgical intervention within the 12 months after the initial permanent CSF diversion. 
Hospitalization costs were defined as per day inpatient costs (including bed costs, maintenance fluids, medications, and bedside care) specifically related to neurosurgical intervention and/or care during the patient's hospitalization for permanent CSF diversion as well as subsequent follow-up visits.

Professional fees were defined as all physician fees associated with the permanent CSF diversion procedures performed during each patient's hospitalization. Apart from a small group of patients whose surgeries were complicated by other underlying medication conditions, the majority of patients had similar, if not the same, professional procedural fees.

For the purposes of this analysis, any temporizing hydrocephalus treatments, including subgaleal shunts, reservoirs, and external ventricular drains, inserted prior to initial permanent CSF diversion were excluded from this analysis. The authors chose to exclude these costs in an effort to effectively compare surgical techniques across etiologies.

Total costs of hospitalization (TCH), for initial surgery and/or revision, for patients undergoing VPS, ETV, or ETV/CPC were calculated as: TCH $=[$ (mean daily hospitalization costs $\times$ length of hospitalization) + mean professional procedural fees]. Furthermore, to avoid over- or underestimating the cost-effectiveness of a particular CSF diversion technique due to small sample sizes in revision groups, we did not utilize "intent-to-treat" methodology. Instead, each revision (independent of type of diversion technique utilized) was considered within the treatment arm of the initial intervention. (Cost breakdowns are further discussed in the Results section and complementing tables.)

\section{TreeAge}

TreeAge (http://www.treeage.com), a healthcare decision and cost-effectiveness analysis program, was used to build a treatment model outlining clinical scenarios and associated costs for the correction of hydrocephalus using VPS, ETV, or ETV/CPC. Additionally, we utilized TreeAge to conduct a 1-way sensitivity analysis to assess the model's vulnerability to cost uncertainties. Models were built to characterize the treatment decision making (VPS, ETV, ETV/CPC) for the initial permanent CSF diversion (Fig. 1). In addition to initial procedures, each model also takes into account subsequent treatment outcomes occurring within 12 months of the initial intervention.

Arms representing a clinical decision-making pathway for each CSF diversion intervention were created. At each outcome point or "terminal node," an incremental effectiveness score was assigned to represent treatment success. For this study, treatment success was defined by the need for additional surgical intervention to address recurring signs and symptoms of hydrocephalus within 12 months after the initial surgical intervention.

Hospitalization costs representing inpatient stays, as well as professional fees for interventions performed, were calculated for the total length of stay in days to represent the total cost associated with that particular CSF intervention and admission. As no specific effectiveness scores have been expanded on in the literature, for purposes of this analysis we utilized historical success/failure rates in our models and assigned arbitrary scores of 1,0.5, and 0.25 , respectively, representing 0,1 , and 2 operative failures. For example, a re-intervention within 12 months of initial intervention requiring a new hospitalization and surgical procedure, contributing to the overall cost of hy-

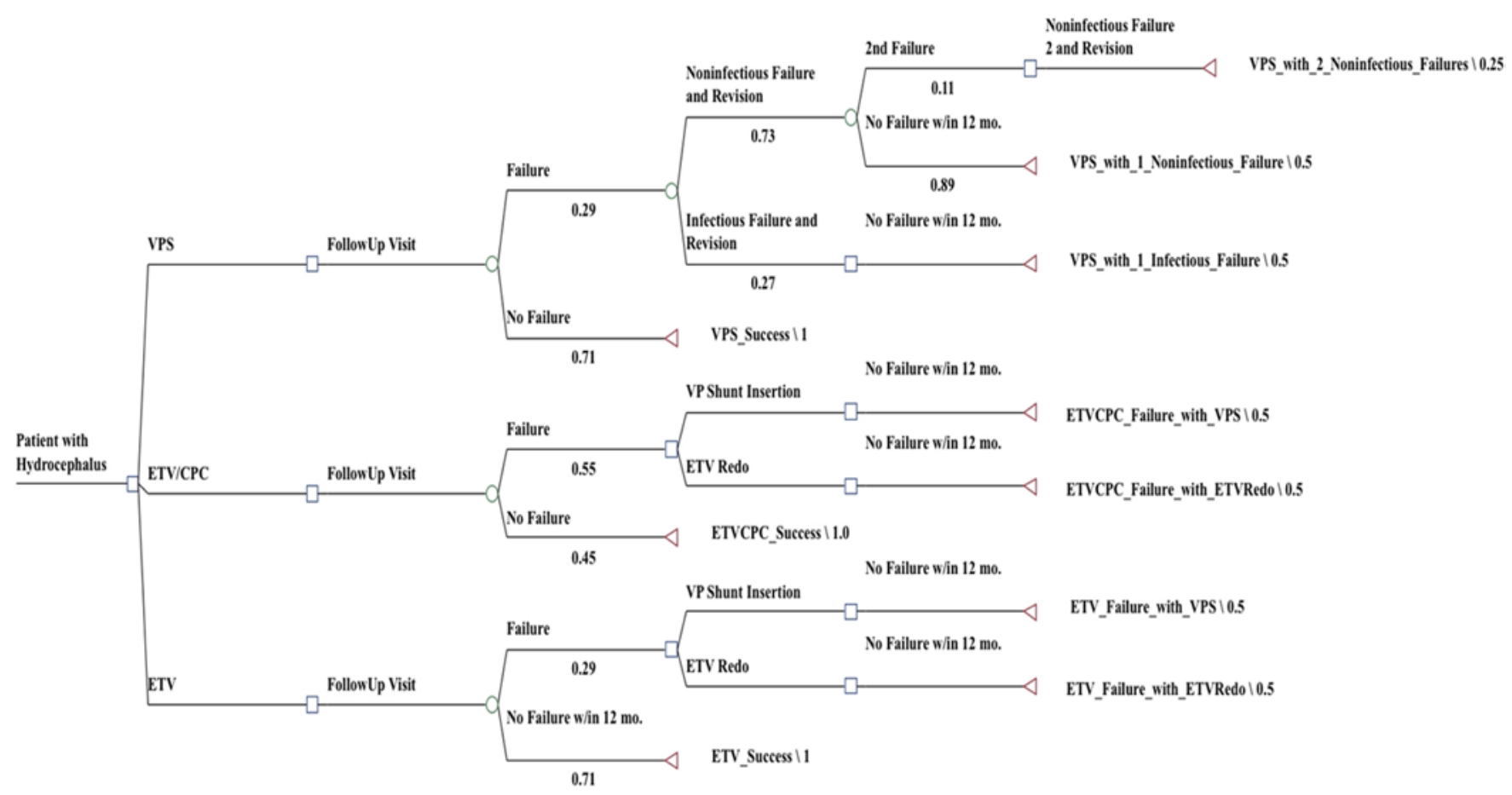

FIG. 1. CSF diversion decision tree. Figure is available in color online only. 
drocephalus treatment and increasing costs 2-fold, making the initial treatment half as effective, would receive an effectiveness score of 0.5 .

Furthermore, for this study, a subjective high willingness-to-pay value was assigned to each model. As this is a study evaluating treatment decisions for a pediatric population, the idea of willingness to pay is somewhat arbitrary for several reasons: 1) the pediatric population is considered a vulnerable population and medical care is rarely, if ever, refused in this setting, and 2) given the current insurance environment, a large portion $(>98 \%$ of our cohort) of the pediatric population is covered by private or public insurance authorization for the procedures. Additionally, although each patient's individual treatment plan allows for consideration with regard to all procedure types, studies have proven that hydrocephalus of certain etiologies responds much better to one procedure than to another, thereby reducing the need for subsequent intervention. Given this scenario, and the previous 2 reasons given, willingness to pay does not often enter into decision making.

We also acknowledge the value of utilizing measures such as disability-adjusted life years (DALYs) and qualityadjusted life years (QALYs), but we determined that these measures of health benefits would not be appropriate for this study (further rationale in the Discussion section). Instead, we assigned arbitrary values based on our historical intervention and re-intervention rates.

\section{Results}

\section{Patient Background}

A total of 147 patients who had undergone CSF diversion were identified. Based on the initial intervention, their cases were classified as follows: 113 VPS cases, 14 ETV cases, and $20 \mathrm{ETV} / \mathrm{CPC}$ cases. The male-female sex ratio of all patients was 1.26 , the total patient cohort was predominantly white $(74.8 \%)$, and the majority of patients were noted to have public insurance. The majority of our cohort fell into 2 etiology categories. In $28.6 \%$ of our cohort, hydrocephalus was secondary to a myelomeningocele diagnosis; the next most frequent etiology was posthemorrhagic hydrocephalus (PHH), present in $13.6 \%$. The remaining cases were categorized into a variety of other etiologies (see Table 1). The mean hospital length of stay of the total patient cohort was 5.0 days. When further evaluating our cohort by etiology, $\mathrm{PHH}$ patients had a mean length of stay of 5.7 days, while patients with myelomeningoceles had a mean length of stay of 3.6 days. The total cost of hospitalization for the cohort was $\$ 77.576 .44$. (See formula in Methods and cost breakdown in Tables 2 and 3.)

\section{Cost by CSF Diversion Intervention}

As the primary outcome of this study we evaluated and compared the length of stay and costs associated with each of the 3 CSF diversion techniques from initial permanent procedures ending with a 12-month post-discharge followup period.

A total of 113 patients underwent initial VPS as a permanent procedure. Of those 113 patients, 22 (19\%) had
TABLE 1. Patient demographics and clinical background

\begin{tabular}{|c|c|c|c|c|}
\hline Variable & $\begin{array}{l}\text { VPS, } \\
n=113\end{array}$ & $\begin{array}{l}\text { ETV } \\
\mathrm{n}=14\end{array}$ & $\begin{array}{l}\text { ETVI } \\
\text { CPC, } \\
n=20\end{array}$ & $\begin{array}{c}\text { Total Study } \\
\text { Cohort, } \\
n=147\end{array}$ \\
\hline \multicolumn{5}{|l|}{ Sex } \\
\hline Male & $67(81.7)$ & $4(4.9)$ & $11(13.4)$ & $82(55.8)$ \\
\hline Female & 46 & 10 & 9 & $65(44.2)$ \\
\hline \multicolumn{5}{|l|}{ Race } \\
\hline White & $84(76.4)$ & $12(10.9)$ & $14(12.7)$ & $110(74.8)$ \\
\hline African American & $21(72.4)$ & $2(6.9)$ & $6(20.7)$ & $29(19.7)$ \\
\hline Asian & $1(100.0)$ & - & - & $1(0.007)$ \\
\hline Native American & $1(100.0)$ & - & - & $1(0.007)$ \\
\hline Other & $6(100.0)$ & - & - & $6(4.1)$ \\
\hline \multicolumn{5}{|l|}{ Insurance } \\
\hline Public & $78(78.0)$ & $7(7.0)$ & $15(15.0)$ & $100(68.0)$ \\
\hline Private & $27(71.1)$ & $7(18.4)$ & $4(10.5)$ & $38(25.9)$ \\
\hline $\begin{array}{l}\text { Government (e.g., } \\
\text { Tricare) }\end{array}$ & $8(88.9)$ & - & $1(11.1)$ & $9(6.1)$ \\
\hline \multicolumn{5}{|l|}{ Hydrocephalus etiology } \\
\hline $\mathrm{PPH}$ & $15(75.0)$ & - & $5(25.0)$ & $20(13.6)$ \\
\hline Myelomeningocele & $33(78.6)$ & - & $9(21.4)$ & $42(28.6)$ \\
\hline \multicolumn{5}{|l|}{ Other etiologies } \\
\hline $\begin{array}{c}\text { Aqueductal } \\
\text { stenosis }\end{array}$ & $2(14.3)$ & $9(64.3)$ & $3(21.4)$ & $14(9.5)$ \\
\hline $\begin{array}{l}\text { Communicating } \\
\text { (congenital) }\end{array}$ & $9(100.0)$ & - & - & $9(6.1)$ \\
\hline Midbrain tumor & $7(87.5)$ & $1(12.5)$ & - & $8(5.4)$ \\
\hline Intracranial cyst & $1(100.0)$ & - & - & $1(0.007)$ \\
\hline Post-head injury & $3(100.0)$ & - & - & $3(2.0)$ \\
\hline Postinfectious & $7(100.0)$ & - & - & $7(4.8)$ \\
\hline $\begin{array}{l}\text { Posterior fossa } \\
\text { cyst }\end{array}$ & $3(100.0)$ & - & - & $3(2.0)$ \\
\hline $\begin{array}{l}\text { Posterior fossa } \\
\text { tumor }\end{array}$ & $10(90.9)$ & $1(9.1)$ & - & $11(7.5)$ \\
\hline $\begin{array}{l}\text { Spontaneous } \\
\text { ICH/IVH/SAH }\end{array}$ & $3(60.0)$ & $1(20.0)$ & $1(20.0)$ & $5(3.4)$ \\
\hline $\begin{array}{l}\text { Supratentorial } \\
\text { tumor }\end{array}$ & $5(100.0)$ & - & - & $5(3.4)$ \\
\hline $\begin{array}{l}\text { Other congenital } \\
\text { anomaly }\end{array}$ & $2(66.7)$ & - & $1(33.3)$ & $3(2.0)$ \\
\hline Other & $13(81.3)$ & $2(12.5)$ & $1(6.3)$ & $16(10.9)$ \\
\hline \multicolumn{5}{|l|}{ NICU stay } \\
\hline Yes & $68(87.2)$ & $2(2.6)$ & $8(10.3)$ & 78 (53.1) \\
\hline No & $45(65.2)$ & $12(17.4)$ & $12(17.4)$ & $69(46.9)$ \\
\hline \multicolumn{5}{|l|}{$\begin{array}{l}\text { No. of readmissions } \\
\text { w/in } 12 \text { mos }\end{array}$} \\
\hline 0 & $78(75.0)$ & $11(10.6)$ & $15(14.4)$ & $104(70.5)$ \\
\hline 1 & $26(78.8)$ & $3(9.1)$ & 4 (12.1) & $33(22.4)$ \\
\hline 2 & $9(90.0)$ & - & $1(10.0)$ & $10(6.8)$ \\
\hline
\end{tabular}

CONTINUED ON PAGE $113 »$ 
» CONTINUED FROM PAGE 112

TABLE 1. Patient demographics and clinical background

\begin{tabular}{lcccc}
\hline \multicolumn{1}{c}{$\begin{array}{c}\text { VPS, } \\
\text { Variable }\end{array}$} & $\begin{array}{c}\text { ETV, } \\
n=113\end{array}$ & $\begin{array}{c}\text { ETVI } \\
\text { CPC, } \\
n=20\end{array}$ & $\begin{array}{c}\text { Total Study } \\
\text { Cohort, } \\
n=147\end{array}$ \\
\hline Comorbidities & & & & \\
\hline Cardiovascular & $28(25)$ & $2(14)$ & $4(20)$ & $34(23)$ \\
\hline Gastrointestinal & $16(14)$ & $1(7)$ & $2(10)$ & $19(13)$ \\
\hline $\begin{array}{l}\text { Hematological \& } \\
\text { immunological }\end{array}$ & $1(0.9)$ & $-(-)$ & $-(-)$ & $1(0.6)$ \\
\hline Malignant & $21(19)$ & $3(21)$ & $-(-)$ & $24(16)$ \\
\hline Metabolic & $-(-)$ & $-(-)$ & $-(-)$ & $-(-)$ \\
\hline Neuromuscular & $82(73)$ & $9(64)$ & $15(75)$ & $106(72)$ \\
\hline Neurosurgical & $44(39)$ & $3(21)$ & $14(70)$ & $61(41)$ \\
\hline Renal & $55(49)$ & $5(36)$ & $9(45)$ & $69(47)$ \\
\hline Respiratory & $7(6)$ & $1(7)$ & $-(-)$ & $8(5)$ \\
\hline Other congenital or & $34(30)$ & $1(7)$ & $3(15)$ & $38(26)$ \\
$\quad$ genetic conditions & & & & \\
\hline
\end{tabular}

$\mathrm{ICH}=$ intracranial hemorrhage; $\mathrm{IVH}=$ intraventricular hemorrhage; $\mathrm{PPH}=$ posthemorrhagic hydrocephalus; $\mathrm{SAH}=$ subarachnoid hemorrhage.

Data are numbers of patients (\%).

operative failure requiring re-intervention, with 20 patients undergoing removal of the pre-existing shunt and insertion of a new VP shunt or VP shunt revision. Only 2 of the 22 patients underwent ETV/CPC as their re-intervention procedure. The mean length of stay for VPS patients was the highest among all initial CSF diversion techniques (5.6 days). Cases of VPS failure were further separated into infectious and noninfectious causes of failure. Those patients diagnosed with a shunt infection had a greater mean length of stay than those requiring a revision for a cause other than infection (13.0 days vs 3.6 days, respectively).
TABLE 3. Total cost of treatment stratified by type of initial intervention

\begin{tabular}{cc}
\hline Initial Treatment \& Outcome & Total Cost \\
\hline VPS & \\
\hline Success & $\$ 88,024.35$ \\
\hline Infectious failure & $\$ 228,783.25$ \\
\hline Noninfectious failure & $\$ 141,843.55$ \\
\hline ETV & $\$ 35,602.27$ \\
\hline Success & $\$ 124,461.32$ \\
\hline New VPS insertion & - \\
\hline ETV redo & $\$ 63,260.80$ \\
\hline ETV/CPC & $\$ 115,420.25$ \\
\hline Success & $\$ 145,159.57$ \\
\hline New VPS insertion
\end{tabular}

Mean total cost for ETV redo after initial ETV procedure could not be calculated as only 1 patient met this criterion. Total cost for this patient was $\$ 159,429.95$.

The group of patients undergoing ETV as their initial CSF diversion procedure had the shortest mean length of hospitalization of the 3 procedure groups (2.36 days). For patients with ETV failure undergoing an insertion of a new VP shunt or undergoing repeat ETV, the mean lengths of hospital stay were 7.3 and 9.5 days, respectively. Patients treated initially with an ETV/CPC had a mean length of hospitalization of 3.35 days. ETV/CPC failures occurred in 8 patients. Seven of these 8 patients underwent additional surgical intervention with insertion of a new VP shunt. The remaining patient underwent a repeat ETV. All ETV/ CPC patients undergoing a revision or repeat procedure had a similar length of stay (range $2.25-4$ days).

The average total cost for initial VPS treatment was $\$ 88,024.35$. Mean total costs were also found to be signifi-

TABLE 2. Total cost of initial intervention stratified by type of CSF diversion

\begin{tabular}{|c|c|c|c|c|}
\hline Intervention Type & Mean Hospitalization Cost Per Day (\$) & Mean Professional Cost $(\$)^{*}$ & Mean LOS (days) & Mean Total Cost $(\$) \dagger$ \\
\hline VPS & $14,838.76$ & $4,244.43$ & 5.65 & $88,024.35$ \\
\hline \multicolumn{5}{|l|}{ VPS failure } \\
\hline Infectious & $10,273.90$ & $7,198.20$ & 13.0 & $140,758.90$ \\
\hline Noninfectious & $13,165.88$ & $6,422.03$ & 3.6 & $53,819.20$ \\
\hline ETV & $13,005.72$ & $4,945.92$ & 2.36 & $35,602.27$ \\
\hline \multicolumn{5}{|l|}{ ETV failure } \\
\hline New VPS insertion & $11,652.35$ & $3,796.89$ & 7.3 & $88,859.05$ \\
\hline ETV redof & $15,703.42$ & $10,247.46$ & 9.5 & - \\
\hline ETV/CPC & $15,300.18$ & $12,005.19$ & 3.35 & $63,260.80$ \\
\hline \multicolumn{5}{|l|}{ ETV/CPC failure } \\
\hline New VPS insertion & $21,091.76$ & $4,703.02$ & 2.25 & $52,159.45$ \\
\hline ETV redo & $17,912.83$ & $10,247.46$ & 4.0 & $81,898.77$ \\
\hline
\end{tabular}

LOS = length of stay.

* Mean professional fee is for total length of stay.

$\dagger$ Mean total cost of intervention $=[($ mean hospitalization cost per day $\times$ mean length of stay $)+($ mean professional cost $)]$.

$\ddagger$ Mean total cost for ETV redo after initial ETV procedure could not be calculated as only 1 patient met this criterion. The total cost for this patient was $\$ 159,429.95$. 


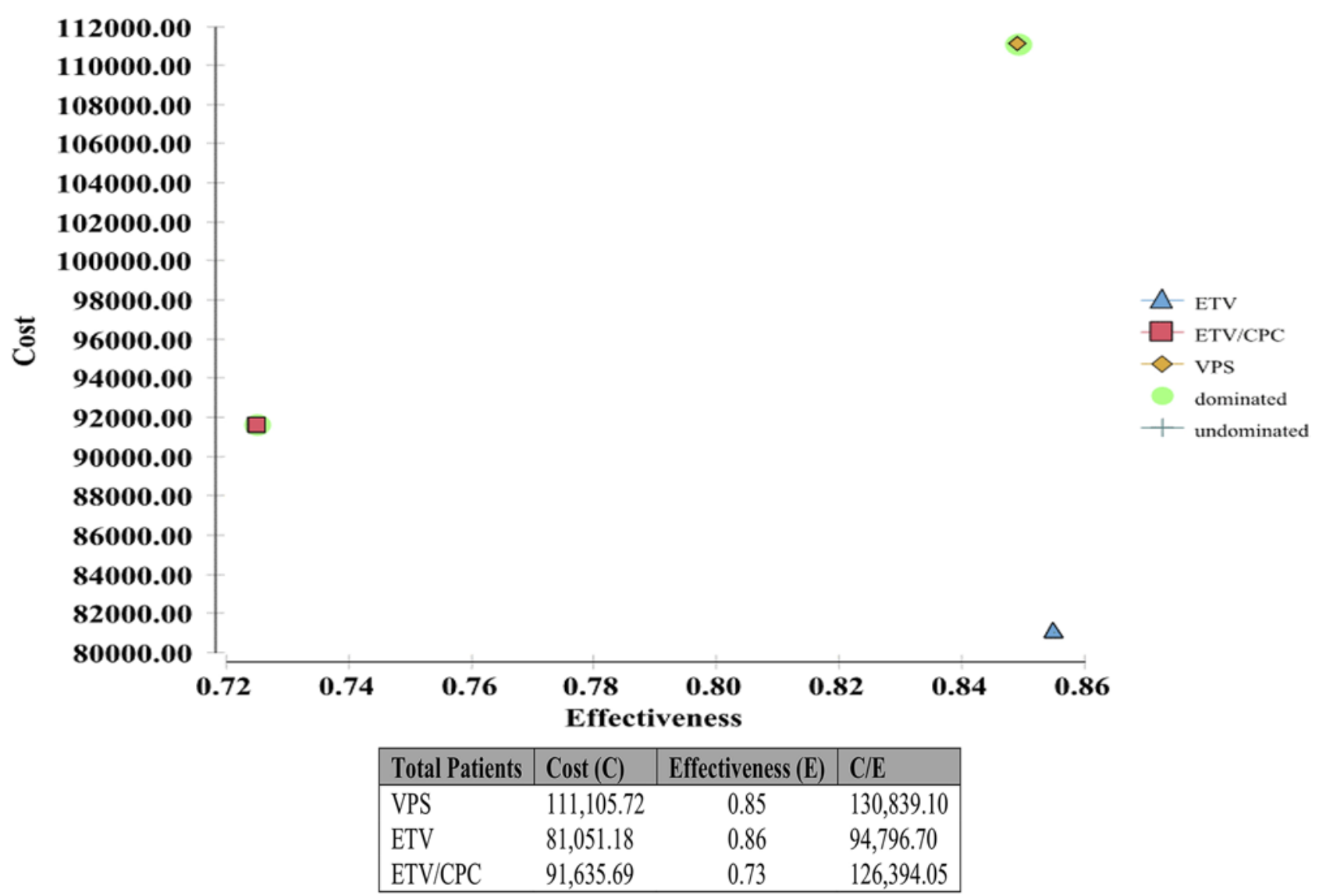

FIG. 2. Cost-effectiveness by CSF diversion. Cost values are in US dollars (US \$). Figure is available in color online only.

cantly different- $\$ 140,758.90$ for infections due to revision and $\$ 53,819.20$ for revisions due to another cause. The average total cost associated with an initial ETV procedure was $\$ 35,602.27$. The average total cost associated with an ETV failure treated with a new VP shunt insertion was $\$ 88,859.05$. Only 1 patient underwent a repeat ETV-at a total cost of $\$ 159,429.95$. ETV/CPC patients incurred an average total cost of $\$ 63,260.80$. This number is higher than that for ETV procedures but lower than that for VPS treatment. When comparing the average costs of ETV/ CPC failures, patients undergoing a new VP shunt insertion procedure had a mean total cost of re-intervention of $\$ 52,159.45$ compared with $\$ 81,898.77$ for patients who underwent a repeat ETV. (See cost breakdown for all procedures in Tables 2 and 3.)

\section{Cost Variation and Cost-Effectiveness of VPS, ETV, and ETV/CPC}

After constructing a cost model representative of the total patient cohort, a cost-effectiveness analysis was conducted using the TreeAge Software. Based on the success and failure rates of VPS, ETV, and ETV/CPC, the associated total costs for the respective surgeries, and the effectiveness score assigned to the different clinical outcomes, a cost-effectiveness analysis report was generated. VPS had an incremental cost-effectiveness ratio (ICER) of $\$ 130,296.14$, which is a measure of the average incremen- tal cost per unit of clinical effectiveness associated with successfully treating hydrocephalus. The ICER for VPS was higher than that for ETV/CPC $(\$ 118,861.76)$. Overall we found that the ICER for ETV $(\$ 75,092.85)$ was lower than for either VPS or ETV/CPC, showing that ETV procedures are less costly in the short run (Fig. 2).

\section{Cost-Effectiveness Comparison by Etiology: Myelomeningocele, $\mathrm{PPH}$, and Other}

We further assessed our data stratified by etiology. In the myelomeningocele patient cohort, VPS was more cost-effective (with an ICER of $\$ 76,620.32$ and clinical effectiveness of 0.85) than ETV/CPC (ICER \$112,762.44, clinical effectiveness 0.78). Similarly, in patients with PHH, VPS was more cost-effective (ICER \$176,866.91 vs $\$ 191,712.61$, respectively) and more clinically effective than ETV/CPC (0.73 vs 0.60). In the combined-etiology cohort excluding myelomeningocele and PHH patients, ETV alone was found to be more cost-effective (ICER $\$ 60,060.99)$ with a clinical-effectiveness score of 0.86 , compared to ETV/CPC (ICER \$93,349.63, clinical effectiveness 0.75 ) and VPS (ICER $\$ 142,134.51$, clinical effectiveness 0.88) (Figs. 3-5).

\section{Sensitivity Analysis}

As our cost-effectiveness model is limited by the sample size from our institution and the short-term follow-up 


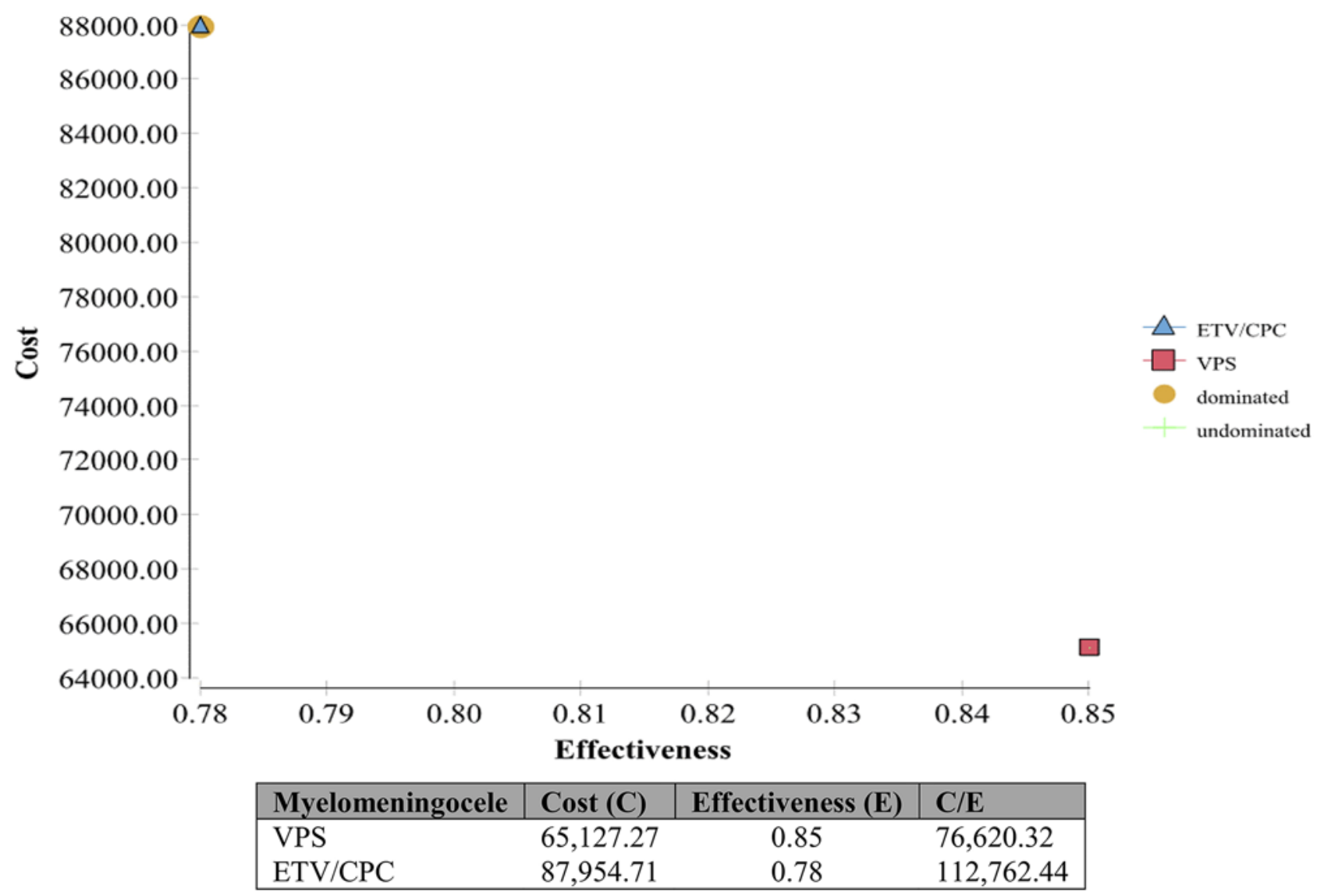

FIG. 3. Cost-effectiveness by myelomingengocele. Cost values are in US dollars (US \$). Figure is available in color online only.

period we conducted a 1-way sensitivity analysis to assess the vulnerability of our model given cost uncertainties. Four independent variables had the potential to impact the model's costs: 1) the daily hospital cost for patients treated with VPS, 2) the average length of hospital stay (in days) after VP shunt placement, 3) the daily cost for patients treated with ETV and CPC (ETV/CPC), and 4) the average length of hospital stay (in days) for patients treated with ETV/CPC. Examination of each allows for estimation of the necessary input value at which ETV/CPC or VPS can surpass ETV as the least expensive option. For VPS to become the least expensive option, the daily hospital costs for VPS patients would have to decrease $56 \%$ from a mean of $\$ 14,839$ to approximately $\$ 6492$, or the mean number of hospital days for patients treated with VPS would need to fall $55 \%$ from a mean of 5.6 to approximately 2.45 days. For ETV/CPC to become the least expensive option, the average hospital daily cost for ETV/CPC patients would need to fall $42 \%$ from a mean of $\$ 15,300$ to approximately $\$ 8798$, or the length of hospital stay would need to decrease $42 \%$ from a mean of 3.4 to approximately 1.96 days. From this analysis, we are confident that ETV remains the lowest-cost option based on the drastic decreases in mean VPS and ETV/CPC inputs needed to reach ETV costs. Of note, these findings do not take clinical effectiveness into account; this analysis was only addressing the economic environment.

\section{Discussion}

The aim of this study was to evaluate the cost of hydrocephalus and through cost-effectiveness methodology gain a better understanding of the variation in surgical intervention costs in an institutional setting such as ours. We were able to gain insight to better appraise treatment decisions from a value-added healthcare perspective. Measured by a cost-effectiveness ratio and under the assumption that a low cost-effectiveness ratio represents the maximum health benefits for a given cost, we determined what permanent CSF diversion procedure allowed for the highest achievable health benefit per cost in dollars for this patient group in the short run..$^{2,20}$

Overall, ETV was found to be less costly and more clinically effective than either VPS or ETV/CPC. When we conducted subgroup analyses by etiology, we found that VPS was more cost-effective and clinically effective than ETV/CPC in both the myelomeningocele and PHH cohorts (no ETV procedures [i.e., ETV without CPC] were performed in these groups during our study period). Also, the calculated net cost associated with VPS was found to be lower than that of ETV/CPC. Thus, VPS yielded a higher aggregate of health benefits than ETV/CPC. This concurs with the literature that establishes VP shunts to be a better treatment for hydrocephalus in the PHH cohort. ${ }^{16}$

When analyzing all other etiologies (combined etiologies), ETV was found to be the value-added procedure of 


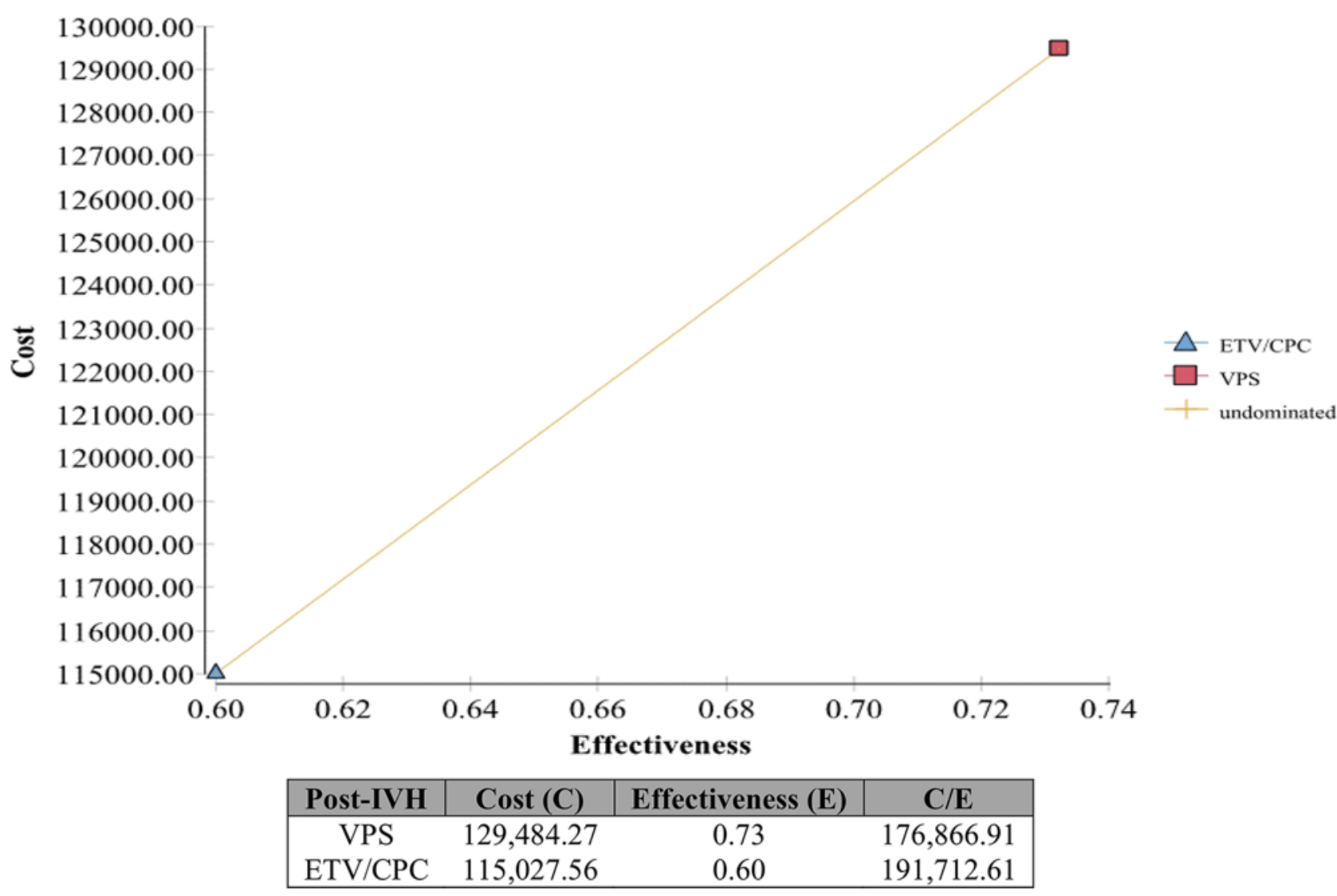

FIG. 4. Cost-effectiveness by PHH. Cost values are in US dollars (US \$). Figure is available in color online only.

choice as it was both cost-effective and clinically effective. The net cost associated with VPS was substantially higher than that associated with ETV or ETV/CPC. The clinical effectiveness of VPS was marginally better than ETV, but given the high costs associated with VPS with the least amount of net yield in clinical benefit, it was a dominant outlier. The ICER values and comparisons for the "other etiologies" patients were very much similar to those for all patients, which makes sense since the "other etiologies" group made up the majority (57.8\%) of the total patient cohort.

VPS was costlier overall due to the longer length of hospitalizations. VPS failures included both noninfectious and infectious etiologies, and VPS infectious failures, as previously reported in the literature, contributed to higher hospital costs due to lengthier hospitalizations, additional treatments including antibiotics, and the need for more laboratory tests, resulting in a higher overall hospitalization cost. The main differences in hospitalization costs between ETV and ETV/CPC were due to the additional costs associated with performing the CPC in addition to the ETV. Our results were further confirmed by our sensitivity analysis. The daily hospitalization costs and average length of stay for VPS and ETV/CPC would require significant reductions in order for either of these procedures to become the most cost-effective. In the days of valueadded healthcare and the burden of clinical effectiveness resting with healthcare systems, we believe there is an opportunity to further identify cost-saving measures that may improve the cost-effectiveness without compromising the clinical effectiveness currently seen.

When comparing our results to those of previously conducted studies we found several differences. First, etiologies of note were different-congenital obstruction and myelomeningocele in the Brazilian study and aqueductal stenosis and tumors in the Canadian study. Second, neither the Brazilian nor the Canadian study found cost differences between ETV and VPS. ${ }^{6,10}$ It is worth noting that the overall cost infrastructure, insurance structure, and patient payment systems of Brazil and Canada greatly differ from those of the United States. The results of cost-effectiveness analyses and the success and failure rates used in our study are etiology- and intervention-dependent. Additionally, success and failure rates utilized were historical representatives of the patient outcomes in our institution. Such aforementioned differences must be taken into consideration when viewing this study's methodologies and conclusions.

For our study, we arbitrarily assigned effectiveness scores to each treatment arm rather than QALYs or DALYs, and this may be both a limitation and strength of the study. As mentioned in the Methods section, there were few to no studies assessing the utility and accuracy of using QALYs in children in the current literature. The 2005 study by Griebsch et al. ${ }^{7}$ was the only identified article that investigated the QALYs in pediatric research. The results of that study showed a wide variation in the definition and use of QALYs from one study to another, and the authors 


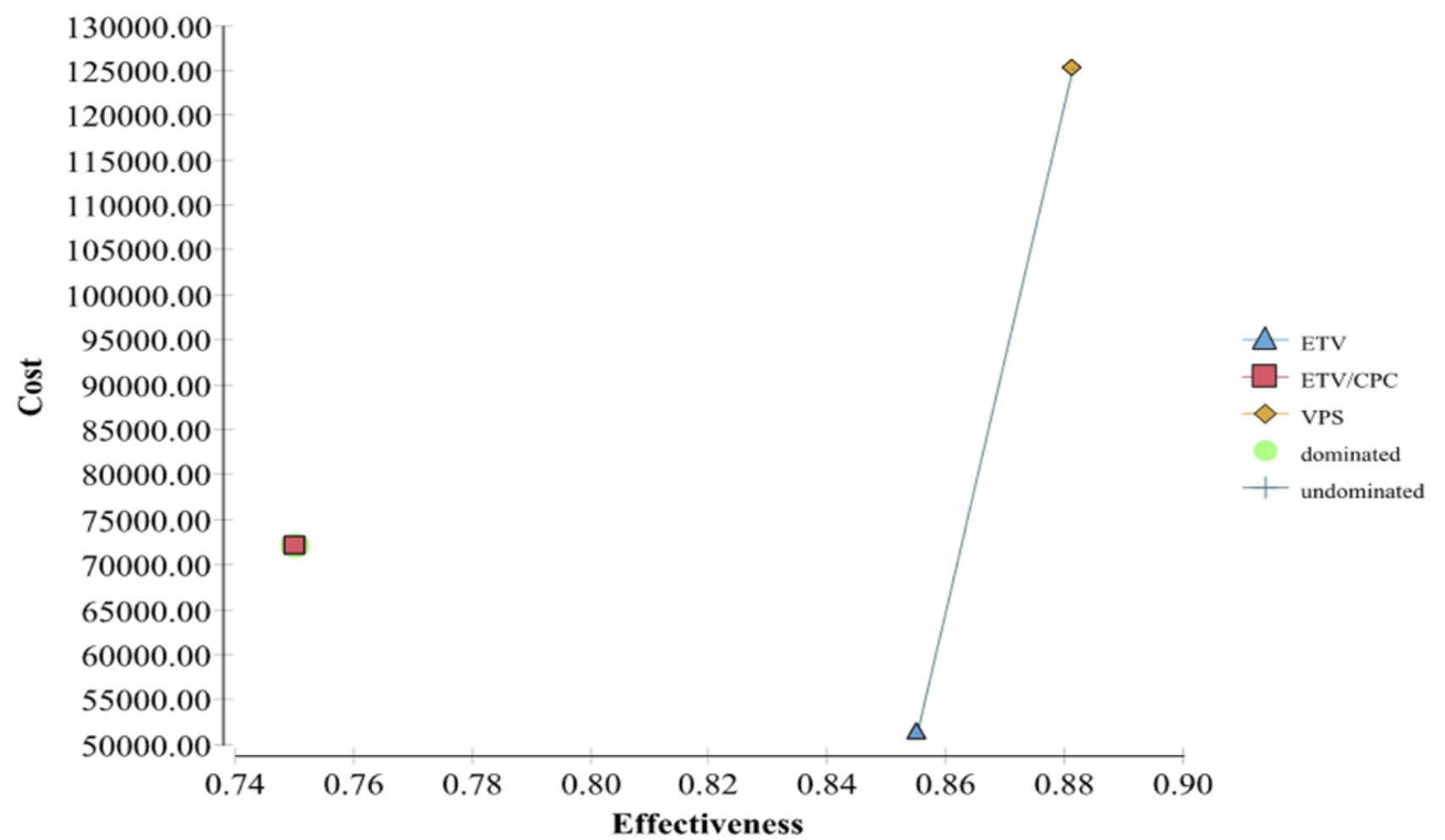

\begin{tabular}{|c|c|c|c|}
\hline Other & Cost (C) & Effectiveness (E) & C/E \\
\hline VPS & $125,252.09$ & 0.88 & $142,134.51$ \\
ETV & $51,352.15$ & 0.86 & $60,060.99$ \\
ETV/CPC & $72,262.22$ & 0.75 & $93,349.63$ \\
\hline
\end{tabular}

FIG. 5. Cost-effectiveness by other etiologies. Cost values are in US dollars (US \$). Figure is available in color online only.

concluded that QALYs may not represent the most optimal outcome measure in children. Children are too young to assign proper QALY scores, and it is often difficult to assess the life year change that a treatment or intervention provides due to the wide variability in child development. Furthermore, the use of DALYs was also considered, but they were not utilized for several reasons: 1) this setting is quite different given the resources immediately available and treatments economically assessable, and 2) the inability to accurately measure or utilized mortality rates in our cohort of patients who have developed hydrocephalus secondary to a variety of etiologies and who have numerous complex chronic conditions. Similar issues have also been previously discussed by Devleesschauwer et al., who noted the difficulty of determining a reliable long-term mortality rate and disease burden in children as a key reason why DALYs were not utilized as a measure of health benefit. ${ }^{3}$ Thus, we decided to use arbitrarily assigned effectiveness scores, which we hope better reflect the efficacy of each treatment arm.

\section{Study Strengths and Limitations}

We recognize several limitations of our study. First, the sample size is small and the patients are from a single institution. Given the failure rates used in this analysis, with power set at 0.80 we would need between 135 (failure rate of 0.29) and 162 (failure rate of 0.55) cases to conduct a real-world cost-effectiveness analysis of each treatment option. Second, the inability to assess the long-term treatment cost-effectiveness or lifetime attributable burden of disease exists. The use of historical institution-specific procedure failure rates and arbitrarily assigned effectiveness scores, instead of well-known QALYs or DALYs, might limit the generalizability of the results. Third, CSF diversion failures can occur later than 12 months after the surgical procedure. However, the majority of failures and subsequent revisions/redos occur during the initial 12-month follow-up period, providing adequate evaluation of the need for re-intervention.

This study is strengthened by the ability to utilize patient-level clinical and financial data specific to the patients we care for at our institution. This method allows us to effectively evaluate our center's clinical decision making and to effectively evaluate the value-added costs associated with caring for our hydrocephalic patient population. We also believe that our models provide a map for other institutions to follow in analyzing their own data.

\section{Conclusions}

This study is one of the first attempts at quantifying the patient-level hospitalization costs associated with surgical management of hydrocephalus in pediatric patients treat- 
ed in the United States. In the short run, ETV was more cost-effective and clinically effective overall. However, VPS was shown to be more cost-effective and clinically effective when we evaluated certain etiologies. Our results indicate that the conversation regarding CSF diversion techniques must be patient-specific and should consider etiology as well as previous surgical intervention. Sensitivity analysis also showed that ETV was the far-superior and more cost-effective procedure. Again, these findings are short-run observations and a long-term follow-up study should be conducted to assess the cost of treating hydrocephalus over the patient's lifetime. We would like to further validate these findings by conducting a cost analysis on a larger scale utilizing multiple institutions, allowing for a greater sample size and more variation in etiology mix.

\section{References}

1. Al-Tamimi YZ, Sinha P, Chumas PD, Crimmins D, Drake J, Kestle J, et al: Ventriculoperitoneal shunt 30-day failure rate: a retrospective international cohort study. Neurosurgery 74:29-34, 2014

2. Centers for Medicare \& Medicaid Services (CMS), HHS: Medicaid and Children's Health Insurance Program (CHIP) programs; Medicaid managed care, CHIP delivered in managed care, and revisions related to third party liability. Final rule. Fed Regist 81:27497-27901, 2016

3. Devleesschauwer B, Havelaar AH, Maertens de Noordhout C, Haagsma JA, Praet N, Dorny P, et al: Calculating disabilityadjusted life years to quantify burden of disease. Int J Public Health 59:565-569, 2014

4. Dewan MC, Lim J, Morgan CD, Gannon SR, Shannon CN, Wellons JC III, et al: Endoscopic third ventriculostomy with choroid plexus cauterization outcome: distinguishing success from failure. J Neurosurg Pediatr 25:655-662, 2016

5. Drake JM, Kulkarni AV, Kestle J: Endoscopic third ventriculostomy versus ventriculoperitoneal shunt in pediatric patients: a decision analysis. Childs Nerv Syst 25:467-472, 2009

6. Garton HJ, Kestle JR, Cochrane DD, Steinbok P: A costeffectiveness analysis of endoscopic third ventriculostomy. Neurosurgery 51:69-78, 2002

7. Griebsch I, Coast J, Brown J: Quality-adjusted life-years lack quality in pediatric care: a critical review of published costutility studies in child health. Pediatrics 115:e600-e614, 2005

8. Harris PA, Taylor R, Thielke R, Payne J, Gonzalez N, Conde JG: Research electronic data capture (REDCap) - a metadata-driven methodology and workflow process for providing translational research informatics support. J Biomed Inform 42:377-381, 2009

9. Kulkarni AV, Riva-Cambrin J, Holubkov R, Browd SR, Cochrane DD, Drake JM, et al: Endoscopic third ventriculostomy in children: prospective, multicenter results from the Hydrocephalus Clinical Research Network. J Neurosurg Pediatr 18:423-429, 2016

10. Lima BO, Pratesi R: Endoscopic third ventriculostomy has no higher costs than ventriculoperitoneal shunt. Arq Neuropsiquiatr 72:524-527, 2014

11. Moorthy RK, Rajshekhar V: Endoscopic third ventriculos- tomy for hydrocephalus: a review of indications, outcomes, and complications. Neurol India 59:848-854, 2011

12. Patwardhan RV, Nanda A: Implanted ventricular shunts in the United States: the billion-dollar-a-year cost of hydrocephalus treatment. Neurosurgery 56:139-145, 2005

13. Scarff JE: Treatment of hydrocephalus: an historical and critical review of methods and results. J Neurol Neurosurg Psychiatry 26:1-26, 1963

14. Scott M, Wycis HT, Murtagh F, Reyes V: Observations on ventricular and lumbar subarachnoid peritoneal shunts in hydrocephalus in infants. J Neurosurg 12:165-175, 1955

15. Shannon CN, Simon TD, Reed GT, Franklin FA, Kirby RS, Kilgore ML, et al: The economic impact of ventriculoperitoneal shunt failure. J Neurosurg Pediatr 8:593-599, 2011

16. Simon TD, Riva-Cambrin J, Srivastava R, Bratton SL, Dean JM, Kestle JR: Hospital care for children with hydrocephalus in the United States: utilization, charges, comorbidities, and deaths. J Neurosurg Pediatr 1:131-137, 2008

17. Stein SC, Guo W: Have we made progress in preventing shunt failure? A critical analysis. J Neurosurg Pediatr 1:40-47, 2008

18. Vinchon M, Rekate H, Kulkarni AV: Pediatric hydrocephalus outcomes: a review. Fluids Barriers CNS 9:18, 2012

19. Warf BC: Endoscopic third ventriculostomy and choroid plexus cauterization for pediatric hydrocephalus. Clin Neurosurg 54:78-82, 2007

20. Weinstein MC, Stason WB: Foundations of cost-effectiveness analysis for health and medical practices. N Engl J Med 296:716-721, 1977

\section{Disclosures}

The authors report no conflict of interest concerning the materials or methods used in this study or the findings specified in this paper.

\section{Author Contributions}

Conception and design: Shannon, Hale, Bonfield, Wellons. Acquisition of data: Lim, Tang, Hysong. Analysis and interpretation of data: Shannon, Lim, Tang, Liles. Drafting the article: Shannon, Lim, Tang, Hysong. Critically revising the article: Shannon, Lim, Liles, Hale. Reviewed submitted version of manuscript: Shannon, Liles. Approved the final version of the manuscript on behalf of all authors: Shannon. Statistical analysis: Shannon, Lim, Liles. Administrative/technical/material support: Shannon. Study supervision: Shannon, Lim, Bonfield, Naftel, Wellons.

\section{Supplemental Information \\ Online-Only Content}

Supplemental material is available with the online version of the article.

Appendix 1. https://thejns.org/doi/suppl/10.3171/2018.6.

\section{PEDS17654.}

\section{Correspondence}

Chevis N. Shannon: Vanderbilt University Medical Center, Nashville, TN. chevis.n.shannon@vanderbilt.edu. 